\title{
Hodgkin's disease in a large thymic cyst in a child
}

\author{
PLC SMITH, C JOBLING, A REES \\ From Harefield Hospital, Harefield, Middlesex
}

Hodgkin's disease of the thymus is well recognised as being associated pathologically with the formation of both microscopic and small macroscopic cysts. ${ }^{1}$ It is, however, unusual for this disease to take the form of small foci of tissue in the wall of a large thymic cyst, as in the patient we report here. The thymic cyst was situated in the left lower hemithorax adjacent to the heart and attached by a narrow pedicle of tissue to the left hilar area.

\section{Case history}

A 14-year-old Caucasian boy presented without symptoms after a routine chest radiograph for tuberculosis screening. This showed a large mass in the left hemithorax related to the heart shadow. Examination showed nothing remarkable except for diminished breath sounds and air entry in the lower left chest.

Routine haematological and biochemical investigations showed a haemoglobin concentration of $12.8 \mathrm{~g} / \mathrm{dl}$, a white blood cell count of $10 \times 10^{9} / 1$, and an erythrocyte sedimentation rate of $25 \mathrm{~mm}$ in one hour. The concentrations of urea, electrolytes, serum albumin, calcium, phosphorus (inorganic), uric acid, and creatinine were all within the normal range of values. Tomography of the left lower hemithorax was performed. This showed a probable cystic mass separate from the heart and attached by a relatively narrow pedicle to the left hilar area. The left upper and lower bronchi could be seen splayed out by the mass.

At left thoracotomy a large cystic mass was found arising from the mediastinum adjacent to the hilum of the left lung and extending to the diaphragm. It was full of turbid, thin fluid. The phrenic nerve ran on the surface of the cyst. The cystic mass was removed with preservation of the phrenic nerve.

Macroscopically the cyst wall showed several areas of nodularity (fig 1). It measured $13 \times 8 \times 6 \mathrm{~cm}$. A microscopic section was reported as showing a combination of simple thymic cyst with Hodgkin's disease tissue of typical nodular sclerotic morphology (fig 2). The Hodgkin's disease was concentrated in the several nodules in the cyst wall. The cyst was lined by epithelium varying in type from non-specific flattened through squamoid to ciliated columnar. Nodular sclerotic Hodgkin's lymphoid aggregates in the wall contained many lacunar cells and Reed-Sternberg cells.

Further investigation showed a normal bone marrow aspirate but computed tomography and lymphography

Address for reprint requests: Mr PLC Smith, Harefield Hospital, Harefield, Middlesex.

Accepted 23 November 1982

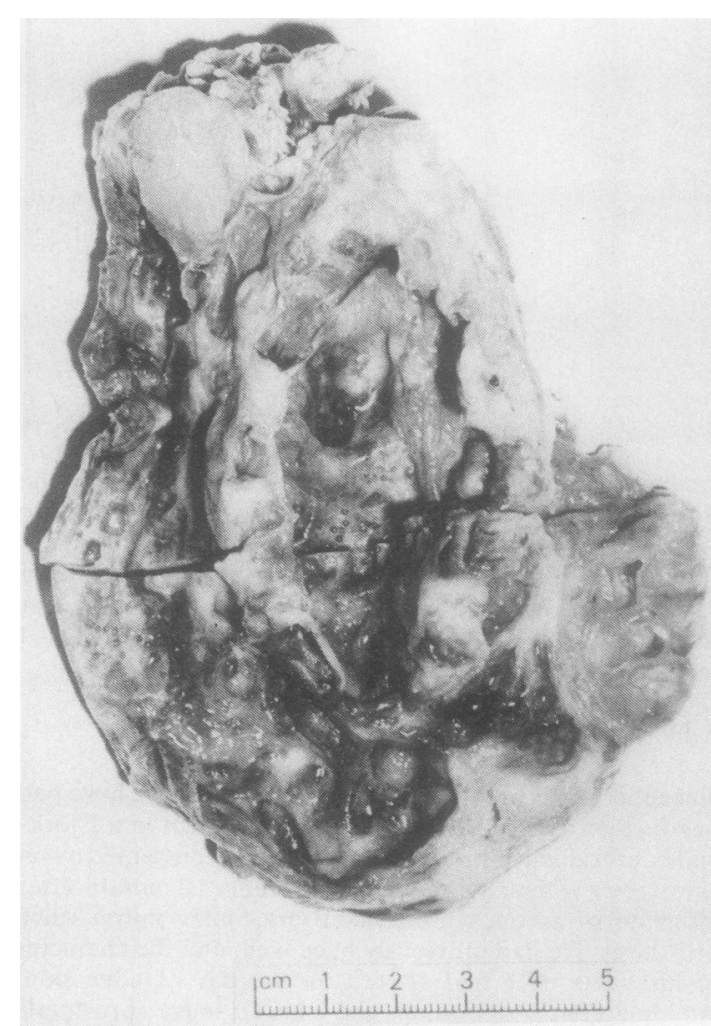

Fig 1 The thymic cyst opened, showing the nodules of tumour tissue in its posterior wall.

showed enlarged iliac and para-aortic lymph nodes with the radiological characteristics of Hodgkin's disease. The $\mathscr{N}$ presence of Hodgkin's disease in the iliac and para-aortic $N$ nodes was not, however, confirmed histologically.

Postoperative recovery was uneventful, the patient later receiving chemotherapy with vinblastine, procarbazine, chlorambucil, and prednisolone.

\section{Discussion}

Hodgkin's disease of the thymus gland is classically of the $\bar{O}$ nodular sclerosing type. It is frequently asymptomatic and $\overrightarrow{\mathbb{D}}$ is found at routine chest radiography, often in a boy or $\frac{\rho}{\Phi}$ young man. The prognosis of the disease is relatively $\varrho$ favourable..$^{1-3}$ It was previously thought that Hodgkin's 


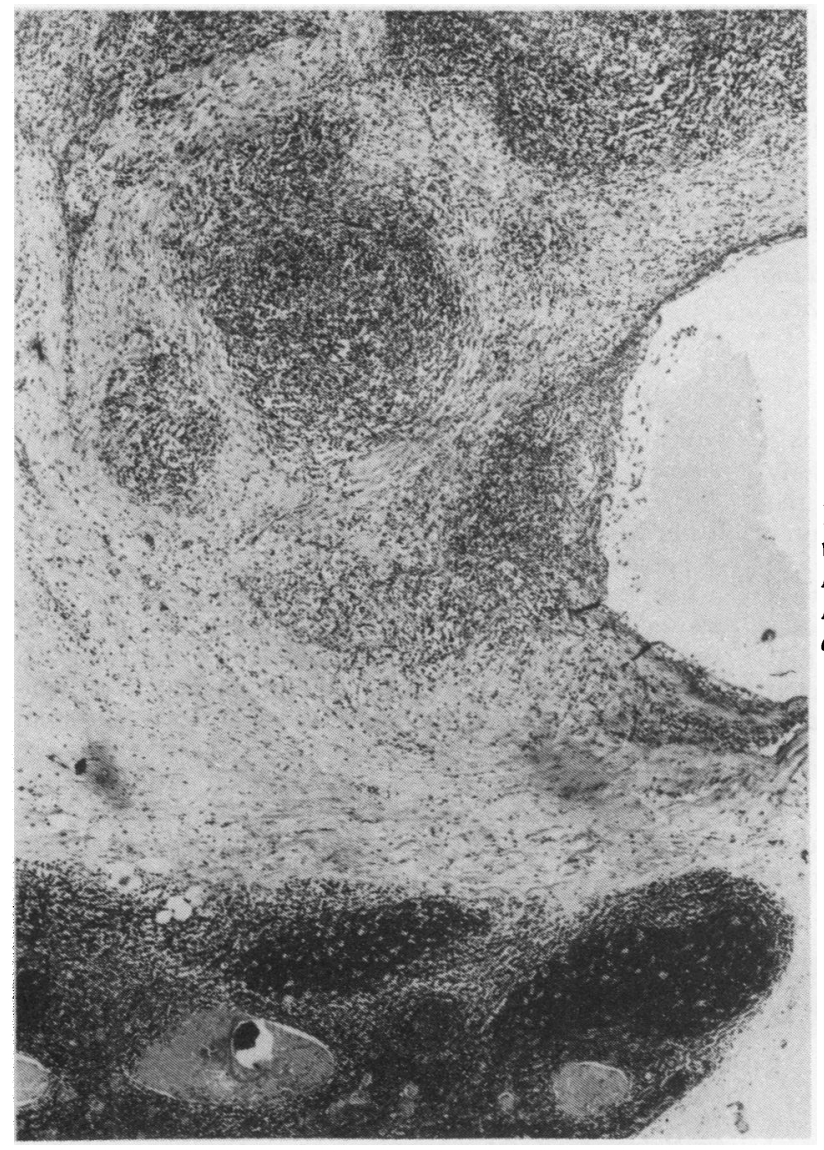

Fig 2 Part of the wall of the cyst; the cystic cavity is seen with typical thymic tissue visible as the darkened mass; the Reed-Sternberg and lacunar cells characteristic of Hodgkin's disease are seen in the central speckled aggregates.

disease of the thymus was in fact à primary thymic neoplasm, often termed granulomatous thymoma. ${ }^{45}$ More recent work, however, has tended to suggest that this is not the case.$^{13}$ Thymic Hodgkin's disease may invade both the lung and the chest wall.

Multiple cysts, both microscopic and macroscopic, are found within the lymphomatous tumour mass. The cysts are lined by thymic epithelium, which is often flattened. It is unusual for the the macroscopic cysts to become large; but this case illustrates the fact that a single cyst may become so large as to form the greater part of the tumour and that the cyst can all but separate from the mediastinum, so that it presents in the pleural cavity. Hodgkin's disease should therefore be considered in the differential diagnosis of any mediastinal cyst, particularly in young male patients.

\section{References}

' Keller AR, Castleman B. Hodgkin's disease of the thymus gland. Cancer 1974;33:1615-23.

2 Burke WA, Burford TH, Donfrian RF. Hodgkin's disease of the mediastinum. Ann Thorac Surg 1967;3:287-96.

${ }^{3}$ Fechner RE. Hodgkin's disease of the thymus. Cancer 1969;23:16.

${ }^{4}$ Lattes-R. Thymoma and other tumours of the thymus. Cancer 1962;15:1224.

s Katz HS, Lattes R. Granulomatous thymoma or Hodgkin's disease of the thymus. Cancer 1969;23:1.

' Eiser NM, Samarrai AR. Thymic lymphoma: an unusual presentation. Thorax 1975;30:588-91. 